Article

\title{
First Report of a Peroxiredoxin Homologue in Jellyfish: Molecular Cloning, Expression and Functional Characterization of CcPrx4 from Cyanea capillata
}

\author{
Zengliang Ruan ${ }^{1, \dagger}$, Guoyan Liu ${ }^{1, \dagger}$, Beilei Wang ${ }^{1, \dagger}$, Yonghong Zhou ${ }^{1}$, Jia Lu ${ }^{1}$, \\ Qianqian Wang ${ }^{1}$, Jie Zhao ${ }^{2, *}$ and Liming Zhang ${ }^{1, *}$
}

1 Department of Marine Biotechnology, Faculty of Naval Medicine, Second Military Medical University, Shanghai 200433, China; E-Mails: ruanzengliang@qq.com (Z.R.); lgy_laurie@aliyun.com (G.L.); lilly_wang@126.com (B.W.); 171006165@qq.com (Y.Z.); cpulj@126.com (J.L.); abc_w@163.com (Q.W.)

2 Department of Chemical Defense Medicine, Faculty of Tropical Medicine and Public Health, Second Military Medical University, Shanghai 200433, China

$\dagger$ These authors contributed equally to this work.

* Authors to whom correspondence should be addressed; E-Mails: zs1010@hotmail.com (J.Z.); lmzhang1969@163.com (L.Z.); Tel.: +86-21-8187-1131 (J.Z.); Tel./Fax: +86-21-8187-1128 (L.Z.).

Received: 19 October 2013; in revised form: 20 December 2013 / Accepted: 23 December 2013 / Published: 9 January 2014

\begin{abstract}
We first identified and characterized a novel peroxiredoxin (Prx), designated as CcPrx4, from the cDNA library of the tentacle of the jellyfish Cyanea capillata. The full-length cDNA sequence of CcPrx 4 consisted of 884 nucleotides with an open reading frame encoding a mature protein of 247 amino acids. It showed a significant homology to peroxiredoxin 4 (Prx4) with the highly conserved F-motif $\left({ }^{93} \mathrm{FTFVCPTEI}^{101}\right)$, hydrophobic region $\left({ }^{217} \mathrm{VCPAGW}^{222}\right),{ }^{140} \mathrm{GGLG}^{143}$ and ${ }^{239} \mathrm{YF}^{240}$, indicating that it should be a new member of the Prx 4 family. The deduced CcPrx 4 protein had a calculated molecular mass of $27.2 \mathrm{kDa}$ and an estimated isoelectric point of 6.3. Quantitative real-time PCR analysis showed that CcPrx 4 mRNA could be detected in all the jellyfish tissues analyzed. CcPrx 4 protein was cloned into the expression vector, pET-24a, and expressed in Escherichia coli Rosetta (DE3) pLysS. Recombinant CcPrx4 protein was purified by HisTrap High Performance chelating column chromatography and analyzed for its biological function. The results showed that the purified recombinant $\mathrm{CcPrx} 4$ protein manifested the ability to reduce hydrogen peroxide and protect supercoiled DNA from oxidative damage,
\end{abstract}


suggesting that CcPrx4 protein may play an important role in protecting jellyfish from oxidative damage.

Keywords: jellyfish; peroxiredoxin; antioxidant; cloning; ROS

\begin{abstract}
Abbreviations
BLAST, Basic Local Alignment Search Tool; CDD, Conserved Domain Database; MEGA, Molecular Evolutionary Genetics Analysis; ROX, Carboxy-X-rhodamine; SYBR, Synergy Brands; $\mathrm{Ct}$, cycle threshold; TOP 10, dh10 $\beta$ Escherichia coli strain; HRP, horse radish peroxidase; G:BOX, Syngene system for fluorescence and visible applications; PDB, Protein Data Bank; YF motif, Tyr-Phe motif.
\end{abstract}

\title{
1. Introduction
}

Exposure to continuous environmental changes, such as solar radiation, pollution, microorganisms, pathogens, salinity and temperature, could lead to the activation of inner defense responses, including the production of reactive oxygen species (ROS) [1]. At low concentrations, ROS may facilitate processes, such as intracellular signaling and defense against microorganisms [2]. However, oxidative stress may occur in the case of excessive production and accumulation of ROS, which would result in a disturbance of metabolic balance, causing damage to cellular lipids, proteins and DNA [3,4]. It is already established that many organisms have both enzymatic and non-enzymatic antioxidant defense mechanisms to minimize such injuries. These antioxidants include various forms of peroxiredoxin, thioredoxin, catalase, glutathione peroxidase and superoxide dismutase [5].

Jellyfish (Scyphozoa) is a class of Cnidaria, which are abundant in pelagic oceanic waters. For the past few years, populations of jellyfish have been exploding in oceans around the world, which has led to many deleterious consequences, threatening human life, fisheries or even ecological balance. On the other hand, however, scientists have also discovered that there are many kinds of highly bioactive substances in the body of jellyfish, which may have good prospects for the development of new marine drugs [6-8]. In particular, as a representative of macroplankton, jellyfish are continuously exposed to harsh environmental factors, such as strong sunlight and ultraviolet (UV) radiation, which may lead to an increase in the production of ROS. Therefore, the exploration of the underlying molecular mechanisms that enable jellyfish to tolerate high levels of oxidative stress could help to better understand the impact of exposure to direct sunlight and UV radiation.

Scientists have isolated some proteins from the jellyfish, Rhopilema esculentum and Stomolophus meleagris, which have strong free radical scavenging abilities and can protect mouse skin lipid and collagen from UV radiation damage $[9,10]$. These results indicate that jellyfish have developed a wide range of powerful antioxidants for self-protection after long-term adaptive selection, suggesting that jellyfish are likely to be a natural resource of antioxidant and anti-UV radiation agents. However, the composition of the antioxidant system in jellyfish species and the sequences, expression levels and bioactivities of some important antioxidant enzymes have not yet been reported until now. 
Peroxiredoxins (Prx proteins) are protective antioxidant enzymes, which are identified as a class of conserved proteins in many organisms, from yeast to mammals [11,12]. It is known that Prx proteins are classified into three types: typical 2-Cys Prx (Prx 1-4), atypical 2-Cys Prx (Prx 5) and 1-Cys Prx (Prx 6). The 2-Cys Prx proteins have two catalytically active Cys residues, termed the peroxidatic and the resolving Cys, whereas the 1-Cys Prx proteins have only the peroxidatic Cys. During the catalytic cycle, the peroxidatic Cys and the resolving Cys form a disulfide bond. In the typical 2-Cys Prx proteins, this bond is intermolecular, whereas in the atypical 2-Cys Prx proteins, it is intramolecular [13-15]. In recent years, the peroxiredoxin superfamily has become one of the hotspots in the research fields of antioxidants, and the most important functions of Prx is considered the scavenging of ROS. These proteins can also act as principal enzymes to regulate the intracellular $\mathrm{H}_{2} \mathrm{O}_{2}$ concentration [16]. Furthermore, Prx has been demonstrated to act as a signal peroxidase to receive, transduce and transmit peroxide signals in mammalian cells [17,18]. In spite of the isolation of Prx genes from numerous organisms, however, the expression and antioxidant function of Prx proteins still remains to be systematically investigated in the jellyfish species.

Cyanea capillata has a worldwide distribution and is one of the common kinds of jellyfish in the Southeast China Sea. In this study, we report a complete Prx cDNA, designated as CcPrx4, from C. capillata. We also characterize its tissue distribution, recombinant protein expression and antioxidant bioactivity in vitro. To our knowledge, this is the first report of a representative antioxidant enzyme from a jellyfish species.

\section{Materials and Methods}

\section{1. cDNA Library Construction}

Total RNA was extracted from the tentacle of $C$. capillata with TRIzol Reagent (Invitrogen, Carlsbad, CA, USA), and then, the mRNA was isolated using the Oligotex mRNA Spin-Column Kit (Qiagen, Valencia, CA, USA). The concentration of purified mRNA was determined at $260 \mathrm{~nm}$ using a BioPhotometer (Eppendorf, Hamburg, Germany). The cDNA library was constructed using the SMART cDNA Library Construction Kit (Clontech, Mountain View, CA, USA), according to the manufacturer's instructions.

\subsection{EST Analysis and Identification of CcPrx4}

EST sequences obtained from the cDNA library of the $C$. capillata tentacle were compared with those in the GenBank database using the BLASTx program to identify genes encoding possible functional proteins. The BLASTx algorithm revealed that one of the EST sequences from the cDNA library showed a significant similarity to protein sequences of the Prx4 family. Thus, this EST sequence was chosen for further analysis and designated as CcPrx4. Subsequently, complete sequencing of both strands of CcPrx 4 cDNA was carried out to confirm that it was a full-length cDNA.

\subsection{Sequence Analysis of the Full-Length CcPrx4 cDNA}

The similarity in nucleotide and protein sequences of CcPrx4 was analyzed using the BLAST algorithm [19,20]. The open reading frame (ORF) was determined using the ORF Finder program [21]. 
A multiple sequence alignment was conducted with the ClustalW2 program [22]. Conserved domains were analyzed using the InterProScan and CDD websites [23-26]. The signal peptide was predicted using the SignalP 4.1 Server [27,28]. The phylogenetic tree was constructed using the neighbor-joining (NJ) method with the MEGA 4 software package. Bootstrap trials were replicated 2000 times. The molecular mass and isoelectric point (pI) was determined using the ProtParam tool [29]. The secondary structure and three-dimensional modeling were predicted using the Phyre2 and SWISS-MODEL algorithms [30-33], respectively. PyMOL (version 0.99rc6 for Windows; Delano Scientific, San Carlos, CA, USA) was used to view and modify the image of the resulting three-dimensional model [34].

\subsection{Quantification Analysis of CcPrx4 Expression by Quantitative Real-Time PCR}

Total RNA was extracted using the UNIQ-10 Kit (Sangon Biotech, Shanghai, China) based on the manufacturer's protocol. Then, single strand cDNA was synthesized according to the manufacturer's instructions using the PrimeScript ${ }^{\circledR}$ RT Reagent Kit (TaKaRa, Otsu, Shiga, Japan), with the total RNA as the template together with Random6 and Oligo (dT) primers. Two gene specific primers, CcPrx4-F (5'-GCCAAGTTTATCCACAAGAGAC-3') and CcPrx4-R (5'-ACTGCTTTTCCTTCCCAATGT-3'), were designed to amplify a product of $103 \mathrm{bp}$. The C. capillata GAPDH gene (GenBank accession number KF595154), used as an internal control, was amplified using the gene specific primers, CcGAPDH-F (5'-GGTGCCCATCAAAACATTATC-3') and CcGAPDH-R (5'-GACACATCAGC AACTGGAACAC-3'), that produced a fragment of $122 \mathrm{bp}$. Quantitative real-time PCR (qRT-PCR) was performed using an ABI PRISM 7300 Sequence Detection System (Applied Biosystems, Foster City, CA, USA). Each reaction (total volume: $25 \mu \mathrm{L}$ ) contained $0.2 \mu \mathrm{M}$ each of the gene specific primers, $0.5 \mu \mathrm{L}$ ROX Reference Dye, $12.5 \mu \mathrm{L} \mathrm{SYBR}^{\circledR}$ Premix Ex Taq ${ }^{\mathrm{TM}}$ and $100 \mathrm{ng}$ cDNA mix as the template, according to the manufacturer's instruction for the SYBR ${ }^{\circledR}$ Premix Ex Taq ${ }^{\mathrm{TM}}$ Kit (TaKaRa, Otsu, Shiga, Japan), and made up to a total reaction volume of $25 \mu \mathrm{L}$ with diethyl pyrocarbonate (DEPC)-treated water. All treatments were performed in triplicate, and data were shown as the mean \pm standard error (SE). The reaction used the thermal profile as follows: $95{ }^{\circ} \mathrm{C}$ for $30 \mathrm{~s}$, followed by 40 cycles of amplification $\left(95^{\circ} \mathrm{C}\right.$ for $15 \mathrm{~s}$ and $60{ }^{\circ} \mathrm{C}$ for $31 \mathrm{~s}$ ). At the end of each PCR reaction, a dissociation curve was obtained by gradual heating of the PCR products from 60 to $95{ }^{\circ} \mathrm{C}$ to confirm that only one PCR product was amplified and detected. For both CcPrx 4 and GAPDH internal control genes, there was only one peak present in the dissociation curves, indicating that the amplifications were specific. Relative gene expression was analyzed by the comparative Ct method ( $2^{-\Delta \Delta \mathrm{Ct}}$ method) with GAPDH as the reference gene, and the results are presented as the relative quantity values [35]. $\mathrm{Ct}$ values for the $\mathrm{CcPrx} 4$ gene were standardized based on those for the GAPDH gene.

\subsection{Construction of the Recombinant Plasmid CcPrx4/pET-24a}

The coding region of CcPrx4 was amplified using standard PCR with the primers, 5'-CTAGCTAGCATGAAAGATGACGAGTC-3' and 5'-CCGCTCGAGCATTTCTTCCTTC-3'. The primers were designed with restriction enzyme sites for Nhe I and Xho I, respectively. The restriction sites are underlined in each primer. The PCR fragment and the pET-24a vector were separately digested with the selected restriction enzymes (NEB, Ipswich, MA, USA); then, the ligation was done at room temperature $\left(25^{\circ} \mathrm{C}\right)$ for $1 \mathrm{~h}$ using T4 DNA ligase (NEB, Ipswich, MA, USA). The ligated 
products were transformed into Escherichia coli TOP 10 competent cells (BioMed, Beijing, China). Positive recombinants were identified using agar plates containing $100 \mu \mathrm{g} / \mathrm{mL}$ kanamycin, followed by nucleotide sequencing of both strands to confirm in-frame insertion.

\subsection{Expression and Purification of Recombinant CcPrx4 Protein in E. coli}

The recombinant plasmid was transformed into the E. coli Rosetta (DE3) pLysS strain for protein expression. Transformed bacteria were propagated at $37{ }^{\circ} \mathrm{C}$ with shaking at $250 \mathrm{rpm}$ in Luria-Bertani (LB) broth containing $100 \mu \mathrm{g} / \mathrm{mL}$ kanamycin and $34 \mu \mathrm{g} / \mathrm{mL}$ chloramphenicol. When the optical density (OD) of the bacteria at $600 \mathrm{~nm}$ reached 0.6 , isopropyl- $\beta$-D-thiogalactoside (IPTG) was added at a final concentration of $0.5 \mathrm{mM}$. Then, the bacteria were shifted to the condition of $12{ }^{\circ} \mathrm{C}$ with shaking at $150 \mathrm{rpm}$ to induce the production of the recombinant protein. After induction for $10 \mathrm{~h}$, the bacteria were subjected to centrifugation at $12,000 \times \mathrm{g}$ for $6 \mathrm{~min}$, and the pellets were collected and resuspended in binding buffer $\left(20 \mathrm{mM} \mathrm{NaH}{ }_{2} \mathrm{PO}_{4}, 500 \mathrm{mM} \mathrm{NaCl}, 30 \mathrm{mM}\right.$ imidazole, $\mathrm{pH}$ 7.4). Subsequently, the resuspended bacterial pellets were lysed by sonication in an ice bath, and the lysate was centrifuged at $12,000 \times \mathrm{g}$ for $30 \mathrm{~min}$ at $4{ }^{\circ} \mathrm{C}$. The supernatant was collected and applied to an ÄKTA protein purification system using a HisTrap High Performance (HP) chelating column (GE Healthcare, Milwaukee, WI, USA). The column was washed with binding buffer $\left(20 \mathrm{mM} \mathrm{NaH} \mathrm{PO}_{4}, 500 \mathrm{mM}\right.$ $\mathrm{NaCl}, 30 \mathrm{mM}$ imidazole, $\mathrm{pH}$ 7.4), and then, the protein of interest was eluted from the column with elution buffer ( $20 \mathrm{mM} \mathrm{NaH} \mathrm{PO}_{4}, 500 \mathrm{mM} \mathrm{NaCl}, 500 \mathrm{mM}$ imidazole, $\mathrm{pH}$ 7.4). Samples collected from different steps were analyzed by $12 \%(w / v)$ sodium dodecyl sulfate-polyacrylamide gel electrophoresis (SDS-PAGE) based on the method of Laemmli, and the gel was stained with Coomassie blue R-250 [36]. Protein concentration was determined according to the method described by Bradford using a standard curve generated with bovine serum albumin (BSA) [37].

\subsection{Western Blotting}

After SDS-PAGE, the proteins in the gel were transferred to a polyvinylidene difluoride membrane (Millipore, Billerica, MA, USA). Subsequently, the membrane was incubated in blocking buffer $5 \%(\mathrm{w} / \mathrm{v})$ fat-free milk powder in Tris-buffered saline and Tween 20 (TBST, containing $50 \mathrm{mM}$ Tris, $150 \mathrm{mM} \mathrm{NaCl}, 0.05 \%(\mathrm{v} / \mathrm{v})$ Tween 20, $\mathrm{pH} 7.6$ ) with gentle shaking for $2 \mathrm{~h}$ at room temperature. It was then incubated with anti-His antibodies from mouse (1:2000 dilution, Tiangen, Beijing, China) at $4{ }^{\circ} \mathrm{C}$ overnight after rinsing the membrane with TBST three times. HRP-labeled goat anti-mouse $\operatorname{IgG}$ (Beyotime, Haimen, Jiangsu, China) diluted to 1:4000 was used as the secondary antibody. The G:BOX system (Syngene, Cambridge, UK) was used for chemiluminescent detection of cross-reacting proteins.

\subsection{In Vitro Peroxidase Activity Assay}

The reaction of Prx catalyzing the reduction of $\mathrm{H}_{2} \mathrm{O}_{2}$ with the presence of dithiothreitol (DTT) has been used to detect in vitro peroxidase activity [38]. The peroxidase activity of the purified CcPrx4 protein was evaluated as previously described, with little modification [39,40]. Briefly, 1-mL reaction mixtures, containing $50 \mathrm{mM}$ 4-(2-hydroxyethyl)-1-piperazineethanesulphonic acid (HEPES) (pH 7.0), $5 \mathrm{mM}$ DTT and the recombinant CcPrx 4 protein or $100 \mu \mathrm{g} / \mathrm{mL}$ heat-inactivated recombinant $\mathrm{CcPrx}$ 
protein (control group), were incubated at room temperature for $10 \mathrm{~min} . \mathrm{H}_{2} \mathrm{O}_{2}$ was added to a final concentration of $100 \mu \mathrm{M}$ to initiate the reactions, and then, they were incubated for $0,2.5,5,7.5$ and $10 \mathrm{~min}$ at room temperature. Subsequently, $100 \mu \mathrm{L}$ of $100 \%(\mathrm{w} / \mathrm{v})$ trichloroacetic acid (TCA) was added to stop the reaction. The mixture was centrifuged to remove the precipitate, followed by the addition of $200 \mu \mathrm{L}$ of $10 \mathrm{mM} \mathrm{Fe}\left(\mathrm{NH}_{4}\right)_{2}\left(\mathrm{SO}_{4}\right)_{2}$ and $100 \mu \mathrm{L}$ of $2.5 \mathrm{M}$ potassium thiocyanate (KSCN), which could react with the remaining $\mathrm{H}_{2} \mathrm{O}_{2}$ and generated the red-colored ferrothiocyanate complex. The remaining amount of $\mathrm{H}_{2} \mathrm{O}_{2}$ in the mixture was estimated by measurement of the red ferrothiocyanate complex. The absorbance was measured at $475 \mathrm{~nm}$. The clearance rate was calculated using the following formula: clearance rate $=\left[\left(\mathrm{A}_{0}-\mathrm{A}_{x}\right) / \mathrm{A}_{0}\right] \times 100 \%$, where $\mathrm{A}_{0}$ was the initial absorbance and $\mathrm{A}_{x}$ was the absorbance after 2.5, 5, 7.5 and $10 \mathrm{~min}$. The assay was performed in triplicate, and data were shown as the mean \pm SE. Statistical analyses were carried out using IBM SPSS Statistics 19. The significance of the difference between each treatment group and the control was analyzed with one-way analysis of variance (ANOVA) and $p$-values lower than 0.05 were considered statistically significant.

\subsection{Metal-Catalyzed Oxidation (MCO) Assay}

The metal-catalyzed oxidation (MCO) assay was conducted to measure the potential of the purified CcPrx4 protein to protect supercoiled DNA against oxidative damage, according to the method described previously with slight modifications [41]. Fifty-microliter reaction mixtures containing $50 \mathrm{mM}$ HEPES (pH 7.0), $35 \mu \mathrm{M} \mathrm{FeCl}_{3}, 10 \mathrm{mM}$ DTT, $1 \mu \mathrm{g}$ supercoiled plasmid DNA of the pET-24a vector and CcPrx4 protein ranging from 25 to $200 \mu \mathrm{g} / \mathrm{mL}$, were incubated at $37{ }^{\circ} \mathrm{C}$ for $2 \mathrm{~h}$. At the end of the incubation, the reaction mixture was subjected to $1 \%(\mathrm{w} / \mathrm{v})$ agarose gel electrophoresis containing Golden View ${ }^{\mathrm{TM}}$ (BioMed, Beijing, China) as the DNA stain to assess the DNA protection effect.

\section{Results}

\subsection{Identification and Sequence Analysis of CcPrx4 cDNA}

An 884 bp full-length cDNA clone was directly isolated from a cDNA library of the C. capillata tentacle by large-scale random sequencing. As shown in Figure 1A, the cDNA contained a 28 bp 5'-untranslated region (UTR), a single open reading frame (ORF) of 741 bp encoding a peptide of 247 amino acids and a 115 bp 3'-UTR, including a stop codon (TAA) and a poly (A) tail. Homology analysis showed that this cDNA had high similarity with members of the Prx4 family. Therefore, the protein was provisionally identified as $\mathrm{CcPrx} 4$ (C. capillata peroxiredoxin4). The calculated molecular mass of the CcPrx 4 protein was $27.2 \mathrm{kDa}$, with an estimated pI of 6.3. Further analysis revealed that the predicted $\mathrm{CcPrx} 4$ protein was a secreted protein, since a predicted $N$-terminal signal peptide with 20 amino acid residues was found in the deduced amino acid sequence. The tertiary structure of the CcPrx 4 protein was also predicted using the Phyre2 program with the Prx4 protein from Homo sapiens (PDB ID: 3TKP, chain B) as the template. This human protein shared $65.0 \%$ identity with the CcPrx4 protein. The CcPrx4 protein consisted of eight alpha-helices and nine beta-strands, and the possible peroxidatic $\mathrm{Cys}^{97}$ was located at the front end of the fourth alpha-helix, while the possible resolving 
$\mathrm{Cys}^{218}$ was located in the middle of the eighth beta-strand (Figure 1B). The complete CcPrx4 cDNA sequence has been submitted to GenBank under the accession number KF201511.

Figure 1. Sequence analysis of CcPrx4. (A) The full-length cDNA nucleotide and deduced amino acid sequences of CcPrx4. The signal peptide is underlined and shaded. The start (ATG) and stop (TAA) codons are bold and underlined. The poly (A) tail is shown bold and italicized at the end of the sequence. (B) The predicted three-dimensional structure of the CcPrx4 protein. Alpha-helices are shown in green, beta-strands in blue and beta-turns in grey. Balls in purple and copper represent $\mathrm{CYS}^{97}$ and $\mathrm{CYS}^{218}$, respectively.
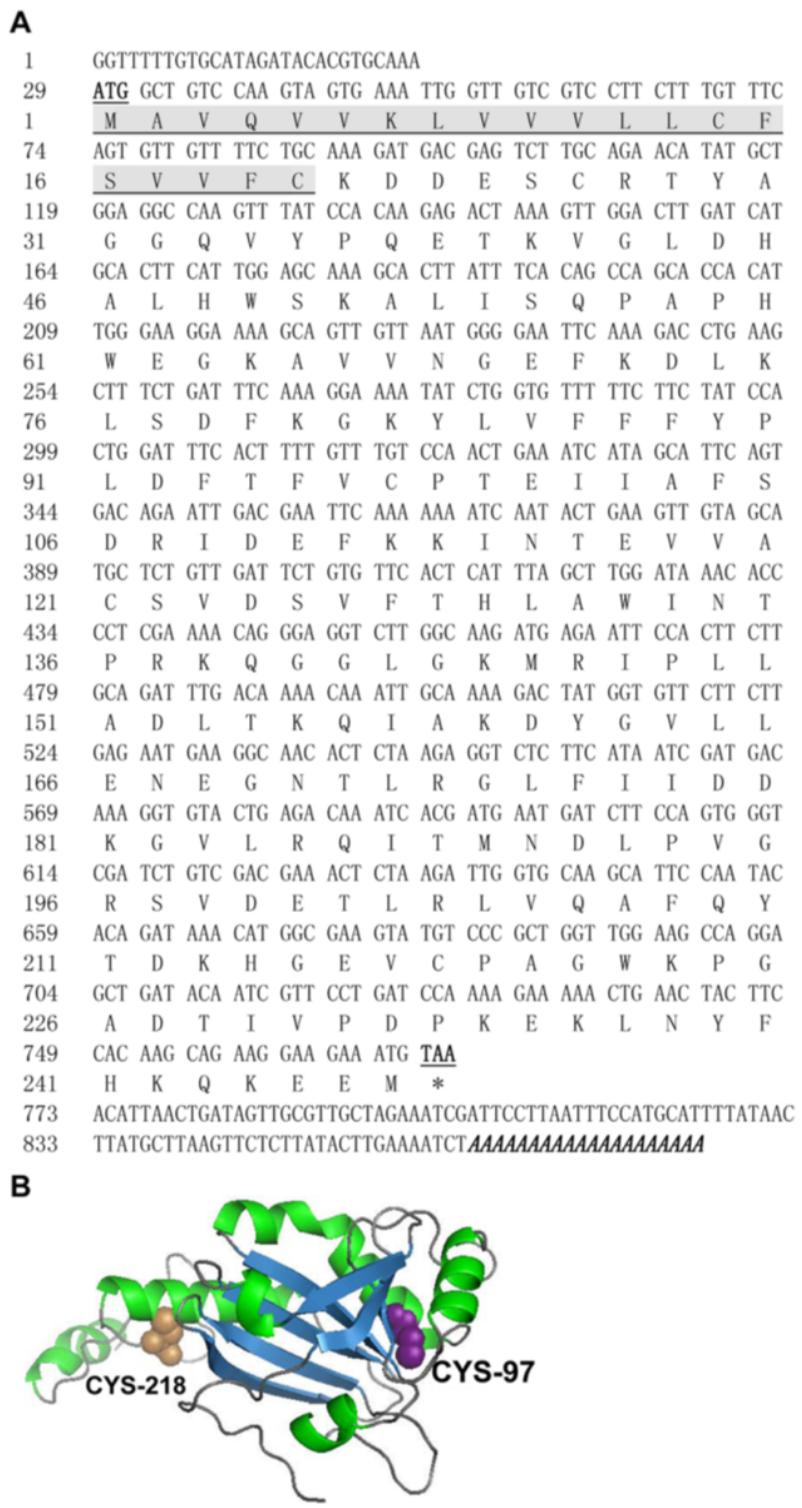

\subsection{Sequence Alignment and Phylogenetic Analysis of the CcPrx4 Protein}

As shown in Figure 2, the predicted amino acid sequence of the CcPrx 4 protein displayed significant homology with other identified Prx4 proteins. The results demonstrated that the F-motif $\left({ }^{93}\right.$ FTFVCPTEI $\left.{ }^{101}\right)$, hydrophobic region $\left({ }^{217} \mathrm{VCPAGW}^{222}\right)$ and ${ }^{140} \mathrm{GGLG}^{143}$ and ${ }^{239} \mathrm{YF}^{240}$ motifs were highly conserved among all the Prx 4 proteins for the species analyzed. The two cysteine-containing 
Table 1. The deduced amino acid sequence of the CcPrx 4 protein compared with the Prx 4 proteins from other species.

\begin{tabular}{cccccc}
\hline Species Name & Common Name & Accession Number & Sequence Size (aa) & Identity (\%) & Similarity (\%) \\
\hline C. capillata & Jellyfish & KF201511 & 247 & - & - \\
H. magnipapillata & Hydra & XP_004207404 & 247 & 74.1 & 81.0 \\
L. salmonis & Sea louse & ACO12581 & 236 & 65.6 & 78.5 \\
M. musculus & Mouse & NP_058044 & 274 & 66.4 & 76.2 \\
R. norvegicus & Norway rat & NP_445964 & 273 & 65.2 & 75.7 \\
H. sapiens & Human & NP_006397 & 271 & 65.0 & 77.0 \\
S. scrofa & Pig & XP_001927404 & 272 & 64.4 & 76.7 \\
X. tropicalis & Western clawed frog & NP_001006812 & 271 & 69.7 & 78.8 \\
S. lalandi & Yellowtail kingfish & ACM47312 & 264 & 68.2 & 77.9 \\
S. salar & Atlantic salmon & ACI69656 & 262 & 68.3 & 77.4 \\
\hline \multicolumn{5}{r}{} &
\end{tabular}

The accession numbers are from the GenBank database.

In order to determine the evolutionary position of the CcPrx4 protein, a phylogenetic tree was constructed (Figure 3). In our phylogenetic tree, the 1-Cys Prx, typical 2-Cys Prx and atypical 2-Cys Prx subgroups were clustered distinctly. CcPrx 4 was positioned in the Prx 4 subgroup and most closely resembled the Prx 4 from H. magnipapillata, which is another marine cnidarian (Class Hydrozoa). This grouping was well-supported by bootstrapping.

Figure 3. Phylogenetic analysis of the deduced amino acid sequence of the CcPrx 4 protein compared with other known Prx proteins in the GenBank database. The number associated with each internal branch was the local bootstrap value, which was an indicator of bootstrap confidence.

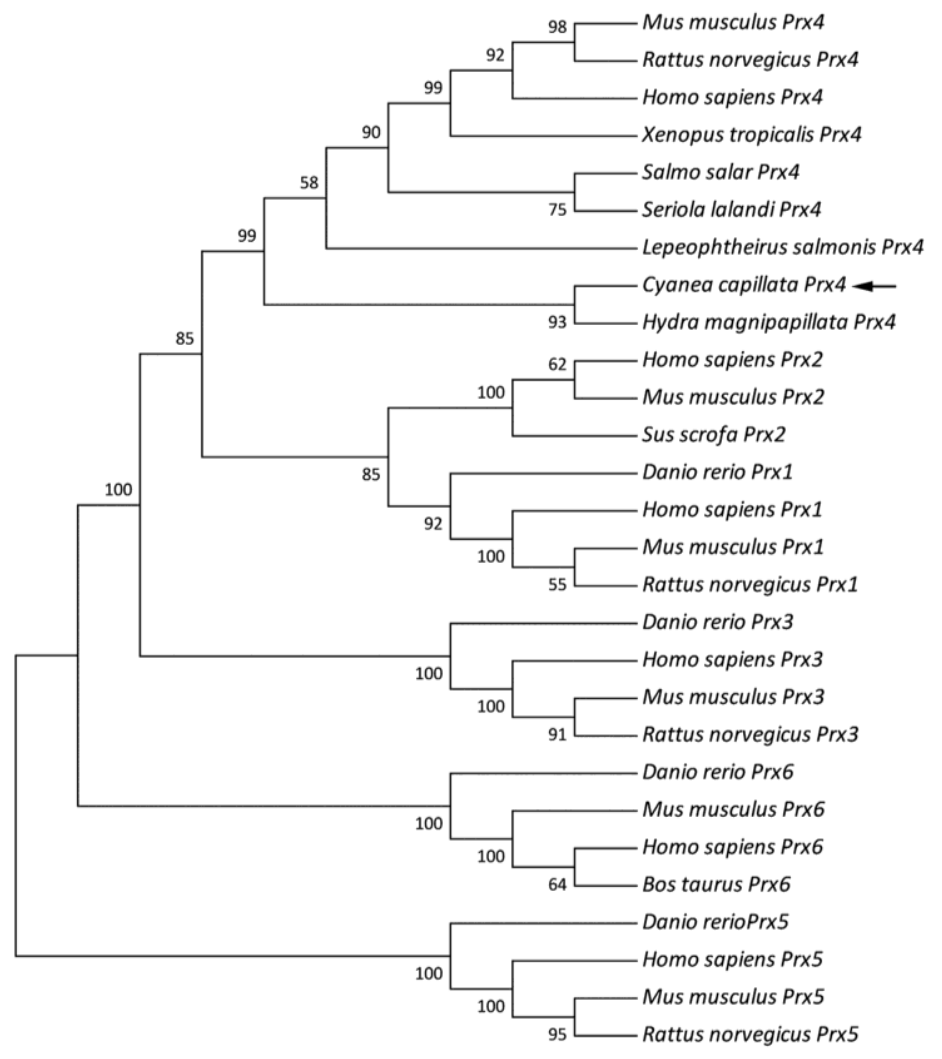




\subsection{Tissue Distribution of CcPrx4}

The CcPrx4 cDNA was originally cloned from a cDNA library made from $C$. capillata tentacle mRNA. Here, we have investigated the expression of CcPrx 4 in other tissues of C. capillata. The relative tissue-specific expression was evaluated by comparing the transcript amount detected in each tissue with that in the tentacle. The results showed that CcPrx 4 mRNA was detectable in all tissues analyzed. The tentacle tissue showed the highest level of expression, followed by the oral arm, the umbrella and, lastly, the gonad (Figure 4).

Figure 4. Tissue distribution of CcPrx 4 mRNA. Relative expression was calculated using the $2^{-\Delta \Delta C T}$ method with GAPDH as the reference gene, and the results are presented as the relative quantity values. All treatments were performed in triplicate, and data were presented as the mean $\pm \operatorname{SE}(n=3)$.

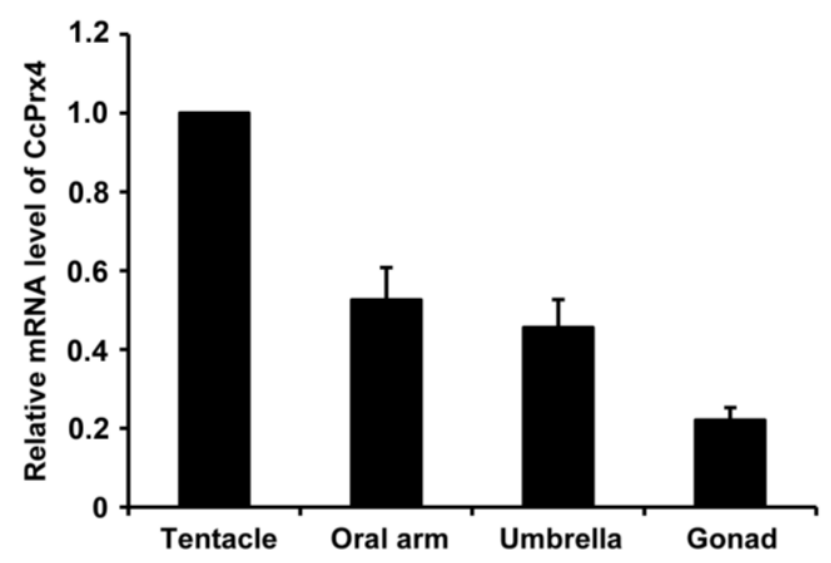

\subsection{Recombinant Expression and Purification of the CcPrx4 Protein}

A CcPrx 4 cDNA encoding the mature protein (not including the signal peptide) was amplified from the $C$. capillata tentacle cDNA library and cloned into the pET-24a expression vector. The encoded protein was expressed in E. coli and then purified using column chromatography. As shown in Figure 5A, there was only one major protein and several very minor proteins that eluted from the column (lanes 4 and 5). The major protein was undetectable in the uninduced cells. The molecular weight $(27.2 \mathrm{kDa})$ of the major protein corresponded well with the expected molecular weight of the recombinant CcPrx4 protein (26.1 kDa without the signal peptide, but with $1.1 \mathrm{kDa}$ of His-Tag). Western blotting analysis using anti-His antibodies further confirmed that the major protein was the His-tagged CcPrx4 fusion protein (Figure 5B). Therefore, it is evident that the CcPrx4 protein was successfully expressed in E. coli and purified to a high level. 
Figure 5. Expression and purification of the recombinant CcPrx 4 protein. (A) $12 \%$ SDS-PAGE analysis of the samples collected from different steps of the expression and purification. Lane 1, whole cell lysates of recombinant E. coli Rosetta (DE3) pLysS before induction; lane 2, whole cell lysates of recombinant E. coli Rosetta (DE3) pLysS after induction with $0.5 \mathrm{mM}$ isopropyl- $\beta$-D-thiogalactoside (IPTG) for $10 \mathrm{~h}$ at $12{ }^{\circ} \mathrm{C}$; lane 3 , fractions from the $30 \mathrm{mM}$ imidazole wash of the HisTrap High Performance (HP) affinity column; lane 4, early fractions from the $500 \mathrm{mM}$ imidazole elution of the HisTrap HP affinity column; lane 5, late fractions from the $500 \mathrm{mM}$ imidazole elution of the HisTrap HP affinity column. The position corresponding to the recombinant CcPrx 4 protein is indicated by an arrow. (B) Western blotting analysis of anti-His antibody cross-reactivity of the proteins separated by SDS-PAGE. The lanes are the same as described for SDS-PAGE in panel A.

A

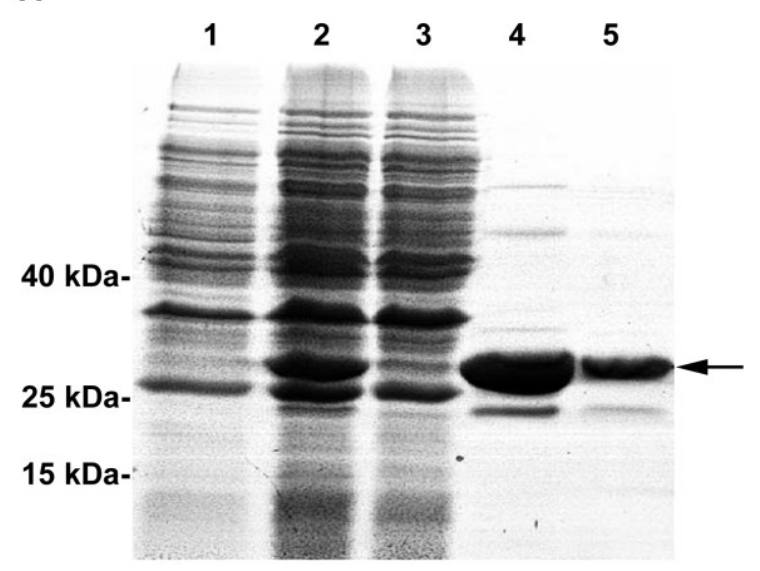

B

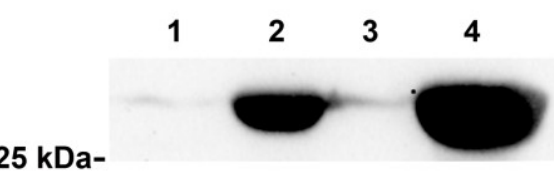

\subsection{In Vitro Peroxidase Activity of the CcPrx4 Protein}

As shown in Figure 6A, only very little degradation of $\mathrm{H}_{2} \mathrm{O}_{2}$ was observed with the heat-inactivated recombinant CcPrx4 protein (control group). However, the CcPrx4 protein distinctly displayed a time- and concentration-dependent activity to reduce $\mathrm{H}_{2} \mathrm{O}_{2}$. At $25 \mu \mathrm{g} / \mathrm{mL}$ of CcPrx4 protein, the clearance rate was slightly increased compared with the control group. When the protein concentration of CcPrx 4 reached 75 and $100 \mu \mathrm{g} / \mathrm{mL}$, the clearance rates were markedly increased to very high levels. It is known that the peroxidase activity of Prx is rapidly inactivated by hydrogen peroxide $[45,46]$. This is what could be occurring in the first $2.5 \mathrm{~min}$ of this assay. Before $2.5 \mathrm{~min}$, the clearance rates were rapidly increased, suggesting that the reactions run very fast during the initial period. After $2.5 \mathrm{~min}$, the reactions have entered a comparatively stationary process. These results suggested that CcPrx 4 protein had a strong efficiency and speed to remove $\mathrm{H}_{2} \mathrm{O}_{2}$, thereby protecting jellyfish against oxidative stress. 
Figure 6. Biological function of the recombinant $\mathrm{CcPrx} 4$ protein. (A) In vitro peroxidase activity of the CcPrx 4 protein in the presence of dithiothreitol (DTT). The clearance rate against reaction time $(\mathrm{min})$ and protein concentration $(\mu \mathrm{g} / \mathrm{mL})$ was monitored to evaluate the $\mathrm{H}_{2} \mathrm{O}_{2}$ reduction activity of $\mathrm{CcPrx} 4$ protein. All treatments were performed in triplicate, and data were shown as the mean $\pm \operatorname{SE}(n=3, * p<0.05 v s$. the control (heat-inactivated), ** $p<0.01$ vs. the control). (B) Effects of CcPrx4 protein in protecting the supercoiled structure of pET-24a plasmid DNA against oxidative damage in the metal-catalyzed oxidation (MCO) system. Lane 1, pET-24a plasmid DNA only; lane 2, pET-24a plasmid DNA and $\mathrm{FeCl}_{3}$; lane 3, pET-24a plasmid DNA and DTT; lane 4, pET-24a plasmid DNA, $\mathrm{FeCl}_{3}$ and DTT; lane 5-10, pET-24a plasmid DNA, $\mathrm{FeCl}_{3}$, DTT and different concentrations of the purified CcPrx4 protein $(200,150,100,75,50$ and $25 \mu \mathrm{g} / \mathrm{mL}$, respectively). The bands corresponding to the nicked form (NF) and the supercoiled form (SF) of the plasmid DNA are indicated on the right-hand side.
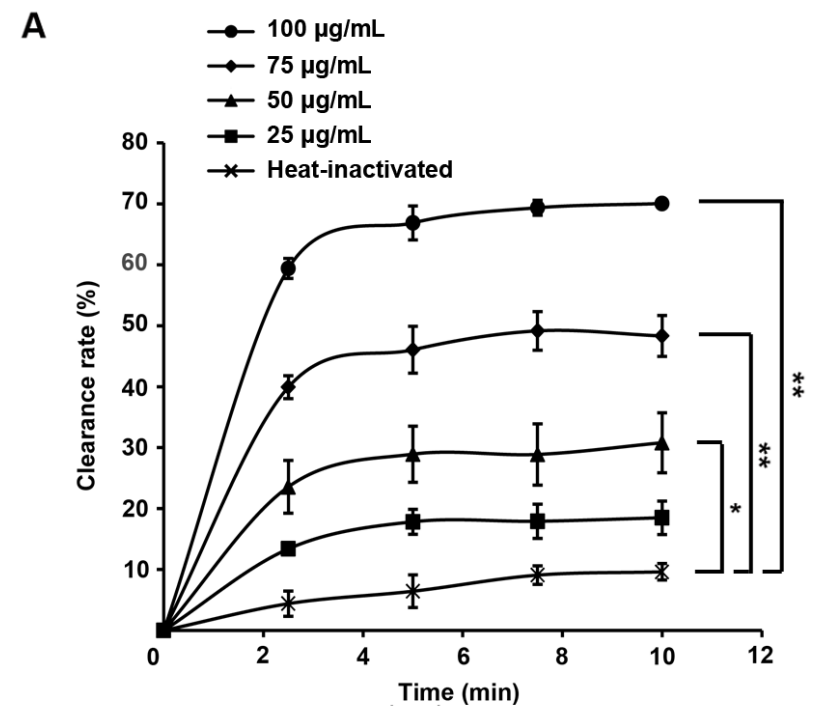

B

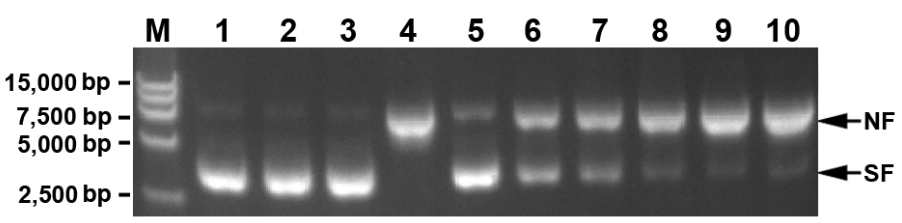

\subsection{Ability of the CcPrx4 Protein to Protect Supercoiled DNA}

The metal-catalyzed oxidation (MCO) assay was performed in order to investigate the ability of CcPrx4 protein to protect the supercoiled structure of DNA against $\mathrm{Fe}^{3+}$ catalyzed oxidative damage [47]. The results showed that the pET-24a plasmid was not damaged by being incubated with $\mathrm{FeCl}_{3}$ or DTT separately at $37{ }^{\circ} \mathrm{C}$ for $2 \mathrm{~h}$, while when incubated with $\mathrm{FeCl}_{3}$ and DTT simultaneously, the plasmid DNA was apparently converted from the supercoiled form to a nicked one. Furthermore, the amount of the nicked form of the plasmid decreased in a dose-dependent manner with increasing concentrations of the recombinant $\mathrm{CcPrx} 4$ protein (Figure 6B). Thus, this is evidence that the CcPrx 4 protein plays a defensive role against DNA damage caused by metal-catalyzed oxidation. 


\section{Discussion}

Jellyfish are members of the phylum, Cnidaria, and naturally distributed in temperate, subtropical and tropical waters. They are typified as free-swimming marine invertebrates consisting of a gelatinous umbrella-shaped bell and trailing tentacles. It has been demonstrated that high light and UV radiation may lead to an increase in the production of ROS, such as superoxide anion, hydrogen peroxide and hydroxyl radicals. The accumulation of these ROSs in cells can cause lipoperoxidation in membranes and DNA damage [48]. Therefore, the extreme conditions in which jellyfish live have attracted our attention, to try to discover the mechanisms that enable them to tolerate high levels of oxidative stress.

C. capillata is an off-shore jellyfish, which has a worldwide distribution and is very common in the coastal waters of southeast China. In the present study, an antioxidant enzyme gene, designated as CcPrx4, was isolated from the cDNA library made from the tentacle of $C$. capillata. Homology analysis showed that this novel molecule had a high similarity with the peroxiredoxin 4 family.

Peroxiredoxin, also called thioredoxin peroxidase (TPx), is a ubiquitous peroxide family, present both in prokaryotes and eukaryotes. It was first discovered as a new type of thiol-specific antioxidant protein from human red blood cells [40]. Many reports have indicated that peroxiredoxin can function to eliminate hydrogen peroxide and rapidly detoxify organic hydroperoxides (ROOH) and peroxynitrite $\left(\mathrm{OONO}^{-}\right)$[49,50]. Therefore, it is thought to be the principal enzyme for the removal of excessive $\mathrm{H}_{2} \mathrm{O}_{2}$ and to protect living organisms from such oxidative damages [51]. Moreover, peroxiredoxin also plays a key role in tumor suppression, immune response, signal transduction, as well as maintaining redox homeostasis [52-58]. Peroxiredoxins do not contain tightly bound metal ions, like other well-known antioxidant enzymes, but they contain highly conserved redox-active cysteine, which is involved in the catalytic mechanism [59].

So far, six kinds of Prx proteins (Prx1-6) have been reported that can be grouped into three categories: 1-Cys, typical 2-Cys and atypical 2-Cys, according to the number of the conserved cysteine residues and the formation of a disulfide bond between the two Cys during its catalytic cycle [13-15]. Multiple sequence alignment showed that the amino acid sequence of the CcPrx 4 protein displayed significant homology with Prx4 proteins from other species, and that it exhibited structural features of the 2-Cys Prx family. The F-motif, including the peroxidatic cysteine, and the hydrophobic region, including the resolving cysteine, were both found in the CcPrx 4 protein sequence, which might be required for its catalytic function, suggesting that this molecule cloned from jellyfish was capable of the same antioxidant attributes as other members of the 2-Cys Prx proteins. Meanwhile, the CcPrx4 protein contains an $\mathrm{NH}_{2}$-terminal hydrophobic region that is typical for the signal sequence of secreting proteins. Actually, Prx4 protein has been considered a secretory protein in mammals [60]. However, there is also a Prx4 protein from black tiger shrimp, Penaeus monodon, which does not possess an $\mathrm{N}$-terminal signal peptide [61]. The phylogenetic tree revealed that $\mathrm{CcPrx} 4$ belonged to a Prx4 branch and most closely resembled the Prx4 from H. magnipapillata, another marine cnidarian. This result could be further supported by the pairwise alignment analysis, where CcPrx4 protein shared the highest identity and similarity with $\operatorname{Prx} 4$ protein from $H$. magnipapillata. Since C. capillata and H. magnipapillata are from the same phylum, they are relatively close to each other in evolutionary history and share similar structures. 
Expression pattern analysis of CcPrx 4 showed that it was found in all the tested jellyfish tissues, including tentacle, umbrella, oral arm and gonad. The ubiquitous expression of the CcPrx 4 protein in jellyfish tissues indicates that it is a critical molecule that may potentially be involved in numerous physiological functions and can act as a very effective antioxidant to remove oxidative stress and protect jellyfish from damage by ROS.

We successfully constructed the recombinant plasmid, CcPrx4/pET-24a, and transformed it into the E. coli Rosetta (DE3) pLysS strain for protein expression and purification. SDS-PAGE and Western blotting results demonstrated that we had obtained the recombinant $\mathrm{CcPrx} 4$ protein with a very high purity. In the assay for in vitro peroxidase activity, the recombinant CcPrx 4 protein could scavenge $\mathrm{H}_{2} \mathrm{O}_{2}$ and exhibited a concentration-dependent peroxidase activity, quickly scavenging $\mathrm{H}_{2} \mathrm{O}_{2}$ in about two minutes. The MCO system has been widely used to assess ROS damage to DNA and has been previously used to assay the antioxidant activity of Prx proteins from different subfamilies [62-64]. In this study, we found that the CcPrx4 protein could protect plasmid DNA from nicking. Moreover, this protective effect was apparently dose-dependent. When the concentration of the recombinant CcPrx 4 protein reached $200 \mu \mathrm{g} / \mathrm{mL}$, the formation of the nicked form was almost completely blocked. All these results indicate that CcPrx4 is a functional homologue of Prx4, which represents a potential protective barrier against oxidative damage in the body of jellyfish. As a result, we propose that the CcPrx 4 protein might be a natural antioxidant and could be developed into the wide applications of food preservatives, sunscreens or drugs for the prevention and treatment of the diseases associated with oxidative stress.

\section{Conclusions}

In conclusion, here, we described the identification, cloning and strong antioxidant activities of a representative antioxidant enzyme from a jellyfish species. Our results strongly support that the CcPrx 4 protein is a key component of the antioxidant system of the jellyfish, C. capillata, which protects the jellyfish body against oxidative exposure. As far as we know, it is the first full-length antioxidant enzyme gene isolated and characterized from jellyfish, which provides a scientific foundation for understanding the mechanism that enables jellyfish to tolerate high levels of oxidative stress and for developing new types of marine drugs.

\section{Acknowledgments}

This work was supported by the Young Scientists Fund of National Natural Science Foundation of China (41306136), the Natural Science Foundation of the Shanghai Municipal Government (12ZR1437000) and the Military Medicine Special-Purpose Foundation of the Second Military Medical University (2012JS04). The authors thank Fang Wei of the College of International Exchange of the Second Military Medical University for his careful revision of the English language of the manuscript.

\section{Conflicts of Interest}

The authors declare no conflict of interest. 


\section{References}

1. Aispuro-Hernandez, E.; Garcia-Orozco, K.D.; Muhlia-Almazan, A.; Del-Toro-Sanchez, L.; Robles-Sanchez, R.M.; Hernandez, J.; Gonzalez-Aguilar, G.; Yepiz-Plascencia, G.; Sotelo-Mundo, R.R. Shrimp thioredoxin is a potent antioxidant protein. Comp. Biochem. Physiol. C Toxicol. Pharmacol. 2008, 148, 94-99.

2. Mates, J.M.; Perez-Gomez, C.; Nunez de Castro, I. Antioxidant enzymes and human diseases. Clin. Biochem. 1999, 32, 595-603.

3. Wang, B.; Zhang, B.; Wang, Q.; Zhang, Z.; Nie, F.; Liu, G.; Zheng, J.; Xiao, L.; Zhang, L. Pharmacological studies of tentacle extract from the jellyfish Cyanea capillata in isolated rat aorta. Mar. Drugs 2013, 11, 3335-3349.

4. Lesser, M.P. Oxidative stress in marine environments: Biochemistry and physiological ecology. Annu. Rev. Physiol. 2006, 68, 253-278.

5. Ataya, F.S.; Fouad, D.; Al-Olayan, E.; Malik, A. Molecular cloning, characterization and predicted structure of a putative copper-zinc SOD from the camel, Camelus dromedarius. Int. J. Mol. Sci. 2012, 13, 879-900.

6. Brinkman, D.; Burnell, J. Identification, cloning and sequencing of two major venom proteins from the box jellyfish, Chironex fleckeri. Toxicon 2007, 50, 850-860.

7. Sanchez-Rodriguez, J.; Torrens, E.; Segura-Puertas, L. Partial purification and characterization of a novel neurotoxin and three cytolysins from box jellyfish (Carybdea marsupialis) nematocyst venom. Arch. Toxicol. 2006, 80, 163-168.

8. Leone, A.; Lecci, R.M.; Durante, M.; Piraino, S. Extract from the zooxanthellate jellyfish Cotylorhiza tuberculata modulates gap junction intercellular communication in human cell cultures. Mar. Drugs 2013, 11, 1728-1762.

9. Li, R.; Yu, H.; Xing, R.; Liu, S.; Qing, Y.; Li, K.; Li, B.; Meng, X.; Cui, J.; Li, P. Isolation, identification and characterization of a novel antioxidant protein from the nematocyst of the jellyfish Stomolophus meleagris. Int. J. Biol. Macromol. 2012, 51, 274-278.

10. Zhuang, Y.; Hou, H.; Zhao, X.; Zhang, Z.; Li, B. Effects of collagen and collagen hydrolysate from jellyfish (Rhopilema esculentum) on mice skin photoaging induced by UV irradiation. J. Food Sci. 2009, 74, H183-H188.

11. Park, S.G.; Cha, M.K.; Jeong, W.; Kim, I.H. Distinct physiological functions of thiol peroxidase isoenzymes in Saccharomyces cerevisiae. J. Biol. Chem. 2000, 275, 5723-5732.

12. Chae, J.I.; Cho, Y.K.; Cho, S.K.; Kim, J.H.; Han, Y.M.; Koo, D.B.; Lee, K.K. Proteomic analysis of pancreas derived from adult cloned pig. Biochem. Biophys. Res. Commun. 2008, 366, 379-387.

13. Dayer, R.; Fischer, B.B.; Eggen, R.I.; Lemaire, S.D. The peroxiredoxin and glutathione peroxidase families in Chlamydomonas reinhardtii. Genetics 2008, 179, 41-57.

14. Seo, M.S.; Kang, S.W.; Kim, K.; Baines, I.C.; Lee, T.H.; Rhee, S.G. Identification of a new type of mammalian peroxiredoxin that forms an intramolecular disulfide as a reaction intermediate. J. Biol. Chem. 2000, 275, 20346-20354.

15. Rhee, S.G.; Chae, H.Z.; Kim, K. Peroxiredoxins: A historical overview and speculative preview of novel mechanisms and emerging concepts in cell signaling. Free Radic. Biol. Med. 2005, 38, $1543-1552$. 
16. Lee, K.S.; Kim, S.R.; Park, N.S.; Kim, I.; Kang, P.D.; Sohn, B.H.; Choi, K.H.; Kang, S. W.; Je, Y.H.; Lee, SM.; et al. Characterization of a silkworm thioredoxin peroxidase that is induced by external temperature stimulus and viral infection. Insect Biochem. Mol. Biol. 2005, 35, 73-84.

17. Randall, L.M.; Ferrer-Sueta, G.; Denicola, A. Peroxiredoxins as preferential targets in $\mathrm{H}_{2} \mathrm{O}_{2}$-induced signaling. Methods Enzymol. 2013, 527, 41-63.

18. Jarvis, R.M.; Hughes, S.M.; Ledgerwood, E.C. Peroxiredoxin 1 functions as a signal peroxidase to receive, transduce, and transmit peroxide signals in mammalian cells. Free Radic. Biol. Med. 2012, 53, 1522-1530.

19. Altschul, S.F.; Gish, W.; Miller, W.; Myers, E.W.; Lipman, D.J. Basic local alignment search tool. J. Mol. Biol. 1990, 215, 403-410.

20. NCBI Basic Local Alignment Search Tool (BLAST) Home Page. Available online: http://www.ncbi.nlm.gov/Blast.cgi (accessed on 13 April 2013).

21. NCBI Open Reading Frame (ORF) Finder Home Page. Available online: http://www.ncbi.nlm.nih.gov/gorf/gorf.html (accessed on 13 April 2013).

22. EMBL-EBI ClustalW2 Home Page. Available online: http://www.ebi.ac.uk/Tools/msa/clustalw2/ (accessed on 13 April 2013).

23. Marchler-Bauer, A.; Anderson, J.B.; Chitsaz, F.; Derbyshire, M.K.; DeWeese-Scott, C.; Fong, J.H.; Geer, L.Y.; Geer, R.C.; Gonzales, N.R.; Gwadz, M.; et al. CDD: Specific functional annotation with the Conserved Domain Database. Nucleic. Acids Res. 2009, 37, D205-D210.

24. Marchler-Bauer, A.; Lu, S.; Anderson, J.B.; Chitsaz, F.; Derbyshire, M.K.; DeWeese-Scott, C.; Fong, J.H.; Geer, L.Y.; Geer, R.C.; Gonzales, N.R.; et al. CDD: A Conserved Domain Database for the functional annotation of proteins. Nucleic. Acids Res. 2011, 39, D225-D229.

25. EMBL-EBI InterProScan 4 Home Page. Available online: http://www.ebi.ac.uk/Tools/ pfa/iprscan/ (accessed on 18 April 2013).

26. NCBI Conserved Domain Database (CDD) Home Page. Available online: http://www.ncbi.nlm.nih.gov/cdd (accessed on 18 April 2013).

27. Petersen, T.N.; Brunak, S.; von Heijne, G.; Nielsen, H. SignalP 4.0: Discriminating signal peptides from transmembrane regions. Nat. Methods 2011, 8, 785-786.

28. CBS SignalP 4.1 Server Home Page. Available online: http://www.cbs.dtu.dk/services/SignalP/ (accessed on 6 May 2013).

29. ExPASy ProtParam Tool Home Page. Available online: http://web.expasy.org/protparam/ (accessed on 6 May 2013).

30. Arnold, K.; Bordoli, L.; Kopp, J.; Schwede, T. The SWISS-MODEL workspace: A web-based environment for protein structure homology modelling. Bioinformatics 2006, 22, 195-201.

31. Kelley, L.A.; Sternberg, M.J. Protein structure prediction on the Web: A case study using the Phyre server. Nat. Protoc. 2009, 4, 363-371.

32. Structural Bioinformatics Group, Imperial College, Phyre 2 Home Page. Available online: http://www.sbg.bio.ic.ac.uk/phyre2/html/page.cgi?id=index (accessed on 30 May 2013).

33. ExPASy SWISS-MODEL Home Page. Available online: http://swissmodel.expasy.org/ (accessed on 30 May 2013). 
34. Bramucci, E.; Paiardini, A.; Bossa, F.; Pascarella, S. PyMod: Sequence similarity searches, multiple sequence-structure alignments, and homology modeling within PyMOL. BMC Bioinforma. 2012, 13, S2:1-S2:6.

35. Livak, K.J.; Schmittgen, T.D. Analysis of relative gene expression data using real-time quantitative PCR and the 2(-Delta Delta C(T)) Method. Methods 2001, 25, 402-408.

36. Laemmli, U.K. Cleavage of structural proteins during the assembly of the head of bacteriophage T4. Nature 1970, 227, 680-685.

37. Bradford, M.M. A rapid and sensitive method for the quantitation of microgram quantities of protein utilizing the principle of protein-dye binding. Anal. Biochem. 1976, 72, 248-254.

38. Pushpamali, W.A.; De Zoysa, M.; Kang, H.S.; Oh, C.H.; Whang, I.; Kim, S.J.; Lee, J. Comparative study of two thioredoxin peroxidases from disk abalone (Haliotis discus discus): cloning, recombinant protein purification, characterization of antioxidant activities and expression analysis. Fish Shellfish Immunol. 2008, 24, 294-307.

39. Lim, Y.S.; Cha, M.K.; Kim, H.K.; Uhm, T.B.; Park, J.W.; Kim, K.; Kim, I.H. Removals of hydrogen peroxide and hydroxyl radical by thiol-specific antioxidant protein as a possible role in vivo. Biochem. Biophys. Res. Commun. 1993, 192, 273-280.

40. Lim, Y.S.; Cha, M.K.; Yun, C.H.; Kim, H.K.; Kim, K.; Kim, I.H. Purification and characterization of thiol-specific antioxidant protein from human red blood cell: a new type of antioxidant protein. Biochem. Biophys. Res. Commun. 1994, 199, 199-206.

41. Li, J.; Zhang, W.B.; Loukas, A.; Lin, R.Y.; Ito, A.; Zhang, L.H.; Jones, M.; McManus, D.P. Functional expression and characterization of Echinococcus granulosus thioredoxin peroxidase suggests a role in protection against oxidative damage. Gene 2004, 326, 157-165.

42. Loo, G.H.; Schuller, K.A. Cloning and functional characterization of a peroxiredoxin 4 from yellowtail kingfish (Seriola lalandi). Comp. Biochem. Physiol. B Biochem. Mol. Biol. 2010, 156, 244-253.

43. Wood, Z.A.; Poole, L.B.; Karplus, P.A. Peroxiredoxin evolution and the regulation of hydrogen peroxide signaling. Science 2003, 300, 650-653.

44. Konig, J.; Lotte, K.; Plessow, R.; Brockhinke, A.; Baier, M.; Dietz, K.J. Reaction mechanism of plant 2-Cys peroxiredoxin: Role of the $\mathrm{C}$ terminus and the quaternary structure. J. Biol. Chem. 2003, 278, 24409-24420.

45. Kil, I.S.; Lee, S.K.; Ryu, K.W.; Woo, H.A.; Hu, M.C.; Bae, S.H.; Rhee, S.G. Feedback control of adrenal steroidogenesis via $\mathrm{H}_{2} \mathrm{O}_{2}$-dependent, reversible inactivation of peroxiredoxin III in mitochondria. Mol. Cell 2012, 46, 584-594.

46. Cho, C.S.; Lee, S.; Lee, G.T.; Woo, H.A.; Choi, E.J.; Rhee, S.G. Irreversible inactivation of glutathione peroxidase 1 and reversible inactivation of peroxiredoxin II by $\mathrm{H}_{2} \mathrm{O}_{2}$ in red blood cells. Antioxid. Redox Signal. 2010, 12, 1235-1246.

47. Suttiprapa, S.; Loukas, A.; Laha, T.; Wongkham, S.; Kaewkes, S.; Gaze, S.; Brindley, P.J.; Sripa, B. Characterization of the antioxidant enzyme, thioredoxin peroxidase, from the carcinogenic human liver fluke, Opisthorchis viverrini. Mol. Biochem. Parasitol. 2008, 160, 116-122.

48. Wang, T.; Wen, X.J.; Mei, X.B.; Wang, Q.Q.; He, Q.; Zheng, J.M.; Zhao, J.; Xiao, L.; Zhang, L.M. Lipid peroxidation is another potential mechanism besides pore-formation underlying hemolysis of tentacle extract from the jellyfish Cyanea capillata. Mar. Drugs 2013, 11, 67-80. 
49. Jeon, S.J.; Ishikawa, K. Characterization of novel hexadecameric thioredoxin peroxidase from Aeropyrum pernix K1. J. Biol. Chem. 2003, 278, 24174-24180.

50. Nelson, K.J.; Knutson, S.T.; Soito, L.; Klomsiri, C.; Poole, L.B.; Fetrow, J.S. Analysis of the peroxiredoxin family: Using active-site structure and sequence information for global classification and residue analysis. Proteins 2011, 79, 947-964.

51. Cheng, X.J.; Yoshihara, E.; Takeuchi, T.; Tachibana, H. Molecular characterization of peroxiredoxin from Entamoeba moshkovskii and a comparison with Entamoeba histolytica. Mol. Biochem. Parasitol. 2004, 138, 195-203.

52. Joung, M.; Yoon, S.; Choi, K.; Kim, J.Y.; Park, W.Y.; Yu, J.R. Characterization of the thioredoxin peroxidase from Cryptosporidium parvum. Exp. Parasitol. 2011, 129, 331-336.

53. Hakimi, H.; Asada, M.; Angeles, J.M.; Inoue, N.; Kawazu, S. Cloning and characterization of Plasmodium vivax thioredoxin peroxidase-1. Parasitol. Res. 2012, 111, 525-529.

54. Henkle-Duhrsen, K.; Kampkotter, A. Antioxidant enzyme families in parasitic nematodes. Mol. Biochem. Parasitol. 2001, 114, 129-142.

55. Fujii, J.; Ikeda, Y. Advances in our understanding of peroxiredoxin, a multifunctional, mammalian redox protein. Redox Rep. 2002, 7, 123-130.

56. Hofmann, B.; Hecht, H.J.; Flohe, L. Peroxiredoxins. Biol. Chem. 2002, 383, 347-364.

57. Dietz, K.J. Plant peroxiredoxins. Annu. Rev. Plant Biol. 2003, 54, 93-107.

58. Wood, Z.A.; Schroder, E.; Robin Harris, J.; Poole, L.B. Structure, mechanism and regulation of peroxiredoxins. Trends Biochem. Sci. 2003, 28, 32-40.

59. McGonigle, S.; Dalton, J.P.; James, E.R. Peroxidoxins: A new antioxidant family. Parasitol. Today 1998, 14, 139-145.

60. Haridas, V.; Ni, J.; Meager, A.; Su, J.; Yu, G.L.; Zhai, Y.; Kyaw, H.; Akama, K.T.; Hu, J.; Van Eldik, L.J.; et al. TRANK, a novel cytokine that activates NF-kappa B and c-Jun $N$-terminal kinase. J. Immunol. 1998, 161, 1-6.

61. Qiu, L.; Ma, Z.; Jiang, S.; Wang, W.; Zhou, F.; Huang, J.; Li, J.; Yang, Q. Molecular cloning and mRNA expression of peroxiredoxin gene in black tiger shrimp (Penaeus monodon). Mol. Biol. Rep. 2010, 37, 2821-2827.

62. Sun, J.; Liu, X.; Li, Q. Molecular cloning, expression and antioxidant activity of a peroxiredoxin 2 homologue from Lampetra japonica. Fish Shellfish Immunol. 2010, 28, 795-801.

63. Shi, G.Q.; Yu, Q.Y.; Shi, L.; Zhang, Z. Molecular cloning and characterization of peroxiredoxin 4 involved in protection against oxidative stress in the silkworm Bombyx mori. Insect Mol. Biol. 2012, 21, 581-592.

64. Yu, F.; Kang, M.; Meng, F.; Guo, X.; Xu, B. Molecular cloning and characterization of a thioredoxin peroxidase gene from Apis cerana cerana. Insect Mol. Biol. 2011, 20, 367-378.

(C) 2014 by the authors; licensee MDPI, Basel, Switzerland. This article is an open access article distributed under the terms and conditions of the Creative Commons Attribution license (http://creativecommons.org/licenses/by/3.0/). 